\title{
Assessment of hair nickel and chromium levels in patients with a fixed orthodontic appliance: a systematic review and meta-analysis of case-control studies
}

\author{
Mohammad Moslem Imani ${ }^{1}$, Mohadeseh Delavarian², Sepideh $\mathrm{Arab}^{3}$, Masoud Sadeghi ${ }^{4}$ \\ 'Department of Orthodontics, Kermanshah University of Medical Sciences, Kermanshah, Iran \\ ${ }^{2}$ Department of Orthodontics, Faculty of Dentistry, Kerman University of Medical Science, Kerman, Iran \\ ${ }^{3}$ Department of Orthodontics, Tehran University of Medical Sciences, Tehran, Iran \\ ${ }^{4}$ Medical Biology Research Center, Kermanshah University of Medical Sciences, Kermanshah, Iran
}

Adv Dermatol Allergol 2019; XXXVI (3): 261-266 DOI: https://doi.org/10.5114/ada.2018.74520

\begin{abstract}
Introduction: The metals nickel $(\mathrm{Ni})$ and chromium $(\mathrm{Cr})$ can cause immunological sensitivity and adverse biological and cytotoxic effects.

Aim: To evaluate hair levels of these metals in patients undergoing fixed orthodontic treatment compared with controls.

Material and methods: Five databases - PubMed, Web of Science, Scopus, Cochrane Library, and ScienceDirect were searched up to January 2018 for evaluation of the hair levels of nickel and/or chromium in patients undergoing fixed orthodontic treatment. To assess the study quality, the Newcastle-Ottawa Scale was used (NOS), and to compare hair $\mathrm{Ni}$ and $\mathrm{Cr}$ levels in the cases compared with the controls, a random-effects meta-analysis was performed by Review Manager 5.3 using standard mean differences (SMDs) and 95\% confidence intervals (Cls).

Results: Out of 38 studies in the databases searched, 6 studies were included in the meta-analysis. The pooled SMD of hair Ni levels between the cases and controls was $0.95 \mu \mathrm{g} / \mathrm{g}(95 \% \mathrm{Cl}:-0.09,1.99 ; p=0.07)$, which showed that the $\mathrm{Ni}$ level was similar in the cases compared with the controls, and that for hair $\mathrm{Cr}$ levels was $0.88 \mu \mathrm{g} / \mathrm{g}(95 \% \mathrm{Cl}$ : $-0.45,2.21 ; p=0.20)$, so the $\mathrm{Cr}$ level was similar in the cases compared with the controls.

Conclusions: The slightly elevated hair levels of $\mathrm{Ni}$ and $\mathrm{Cr}$ in patients undergoing fixed orthodontic treatment suggest that changing the components of fixed orthodontic appliances can be considered as an acceptable solution in the future.
\end{abstract}

Key words: fixed orthodontic treatment, nickel, chromium, hair.

\section{Introduction}

Fixed orthodontic appliance therapy for a mean period of 16 months can lead to increased levels of nickel (Ni) and chromium $(\mathrm{Cr})$ ions in the saliva of patients [1], which can be an uncomfortable experience and affect the quality of life [2]. Ni and $\mathrm{Cr}$ are metals commonly used in the structure of fixed orthodontic appliances, including bands, brackets, and wires [3,4]. Nickel is the most common contact allergen $[5,6]$ affecting women in Europe and the USA [6]. Ni, as an allergen, can cause immunological sensitivity in a number of people receiving long-term orthodontics. The Cr ion can also cause derma- titis, asthma and adverse biological and cytotoxic effects [7]. Mineral analysis of hair is accepted as a meaningful indicator of chronic exposure to and ingestion of metals such as $\mathrm{Ni}$ [5], and the content of nickel in hair and nails correlates with that of blood [8, 9]. Analysis of elements in hair tissue provides a noninvasive biomarker of exposure [10]. Although $\mathrm{Ni}$ and $\mathrm{Cr}$ are known as allergenic and cytotoxic orthodontic metals, very few and controversial studies have assessed the effect of orthodontic treatment on their systemic levels reflected by their best biomarker of exposure, hair [11].

Address for correspondence: Masoud Sadeghi, Medical Biology Research Center, Kermanshah University of Medical Sciences, 67145-1673 Kermanshah, Iran, phone: +98 9188566877, e-mail: sadeghi_mbrc@yahoo.com Received: 22.01.2018, accepted: 19.02.2018. 


\section{Aim}

The aim of this meta-analysis was to investigate hair $\mathrm{Ni}$ and $\mathrm{Cr}$ levels in subjects undergoing fixed orthodontic treatment compared with controls without orthodontic treatment.

\section{Material and methods}

\section{Search strategy and study selection}

The meta-analysis was designed based on the guidelines for the Preferred Reporting Items for Systematic Reviews and Meta-Analyses (PRISMA) [12]. A comprehensive search was done using the databases PubMed, Web of Science, Scopus, Cochrane Library, and ScienceDirect from 1983 to January 2018. We searched the databases by a combination of the terms "nickel", "chromium", "orthodontic" and "hair". The studies were selected for assessment of the hair levels of nickel and/or chromium in patients undergoing fixed orthodontic treatment (cases) compared with controls in the English abstract. The studies included in the meta-analysis had to: (I) use a case-control design, (II) report $\mathrm{Ni}$ and/or Cr levels after orthodontic treatment, (III) report controls without orthodontic treatment, and (IV) report the fixed appliances (archwires, brackets, tubes and bands) with stainless steel and/or Ni-Ti materials. There were no exclusion criteria for the case-control studies included.

\section{Data extraction}

One author (M.S.) searched and two authors (M.S. \& M.M.I.) reviewed independently the articles for relevant publications. We used a standard protocol and reporting form for data extraction from each publication including the first author's name, the year of publication, the country in which the study was conducted, the number of the cases and controls, the duration of treatment, and the mean age/gender (\%) of the cases and controls.

\section{Quality assessment}

To evaluate the study quality, a nine-star protocol was used on the basis of the Newcastle-Ottawa Scale (NOS) for case-control studies [13]. A high-quality study was a study with $\geq 7$ stars.

\section{Statistical analysis}

To compare hair $\mathrm{Ni}$ and $\mathrm{Cr}$ levels in the cases compared with the controls, a random-effects meta-analysis was performed by Review Manager 5.3 (RevMan 5.3, The Cochrane Collaboration, Oxford, United Kingdom) using standard mean differences (SMDs) and 95\% confidence intervals (Cls). Because the orthodontic treatment periods were different in the studies, we used the SMD. Heterogeneity among studies was evaluated with the $Q$ and the $l^{2}$ statistics. The results were defined as heteroge- neous for $p<0.10$ or $R^{2}>50 \%$ [14] and a $p$-value (2-sided) $<0.05$ was considered statistically significant in this meta-analysis. Sensitivity analyses were further conducted in which one study was removed and the rest were analyzed to evaluate whether the results were affected statistically significantly. In addition, the publication bias was assessed by visual inspection of funnel plots and formal testing using Begg's and Egger's tests [15]. The units of measurement of hair nickel or chromium levels were $\mu \mathrm{g} / \mathrm{g}$ dry hair mass.

\section{Results}

\section{Literature search and study characteristics}

Out of 38 studies in the databases searched, after removing duplication studies, 18 studies were screened (Figure 1). Nine studies were excluded because they did not have relevant data and therefore the full texts of 9 studies were evaluated for eligibility. Then, 3 studies were excluded with reasons (2 studies were cross-sectional, and 1 study had two intervention groups without a control). Finally, 6 studies were included in the metaanalysis.

Table 1 shows the characteristics of the 6 studies included in the meta-analysis. The studies were published from 2006 to 2018. Two studies were reported from Poland [16, 17], 2 studies from Iran [3, 5], 1 study from Spain [18], and 1 study from Italy [19]. The study included 197 cases and 173 controls. The orthodontic treatment period was from 4 to $~ 69.8$ months.

\section{Standard mean differences of $\mathrm{Ni}$ and $\mathrm{Cr}$ levels between cases and controls}

The pooled SMD of hair Ni levels between the cases and controls was $0.95 \mu \mathrm{g} / \mathrm{g}(95 \% \mathrm{Cl}:-0.09,1.99 ; p=0.07$; $\left.R^{2}=95 \% ; p_{\text {heterogeneity }}<0.00001\right)$; the result showed that the Ni level was similar in the cases compared with the controls, but with a slightly elevated level in the cases (Figure 2). To reduce heterogeneity (sensitivity analysis), we removed 1 study [3] from the analysis so that pooled SMD became $0.04 \mu \mathrm{g} / \mathrm{g}(95 \% \mathrm{Cl}:-0.41,0.49 ; p=0.87$; $R^{2}=72 \%$; $p_{\text {heterogeneity }}=0.006$ ); the result showed again that the Ni level was similar in the cases compared with the controls.

The result of the pooled SMD of hair $\mathrm{Cr}$ levels between the cases and controls was $0.88 \mu \mathrm{g} / \mathrm{g}(95 \% \mathrm{Cl}$ : $-0.45,2.21 ; p=0.20 ; l^{2}=96 \% ; p_{\text {heterogeneity }}<0.00001$ ). Therefore, the Cr level was similar in the cases compared with the controls, but with a slightly elevated level in the cases (Figure 3). To reduce heterogeneity (sensitivity analysis), we removed 1 study [3] from the analysis so that the pooled SMD became $-0.16 \mathrm{\mu g} / \mathrm{g}(95 \% \mathrm{Cl}:-0.45$, $0.13 ; p=0.28 ; I^{2}=18 \% ; p_{\text {heterogeneity }}=0.30$ ); the result showed that the $\mathrm{Cr}$ level was similar in the cases compared with the controls. 


\section{Quality assessment}

Study-specific quality scores are summarized in Table 2. The mean score was 7. Four case-control studies were awarded $\geq 7$ stars and defined as high-quality studies.

\section{Publication bias}

Figure 4 shows the funnel plot of studies included in two analyses. Begg's test did not reveal, but Egger's test did reveal, significant evidence of publication bias among the included studies (Figure $4 \mathrm{~A}$ ) for reporting $\mathrm{Ni}$ level, whereas Begg's and Egger's tests did not reveal significant evidence of publication bias among the included studies based on sensitivity analysis and removing one study [3]. Begg's and Egger's tests did not reveal significant evidence of publication bias among the included studies for reporting $\mathrm{Cr}$ level (Figure 4 B) and also among the included studies based on sensitivity analysis and removing one study [3].

\section{Discussion}

Corrosion of orthodontic appliances and metal ion release in the environment of the oral cavity are recognized as two main factors for metal ion release [17]. This meta-analysis evaluated hair $\mathrm{Ni}$ and $\mathrm{Cr}$ levels in subjects undergoing orthodontic treatment compared with controls. The results showed that levels of both metals were higher in the patients, but these differences were not significant even with regard to sensitivity analysis. In addition, the increase in Ni level was greater than the increase in $\mathrm{Cr}$ level, when compared to the controls. Out of six studies in the meta-analysis including hair Ni levels, three studies $[3,5,16]$ showed higher hair levels of $\mathrm{Ni}$ in the patients and the other studies [17-19] showed lower levels. Also, out of five studies including $\mathrm{Cr}$ levels, two studies $[3,16]$ reported higher hair levels of $\mathrm{Ni}$ of the cases and the other studies $[17,18]$ reported lower levels. Among all studies, only the study of Jamshidi et al. [3] showed significant differences in $\mathrm{Ni}$ and $\mathrm{Cr}$ concentrations between the two groups and reported that due to the slightly elevated levels of $\mathrm{Ni}$ and $\mathrm{Cr}$ ions in hair of patients undergoing fixed orthodontic treatment the cy-

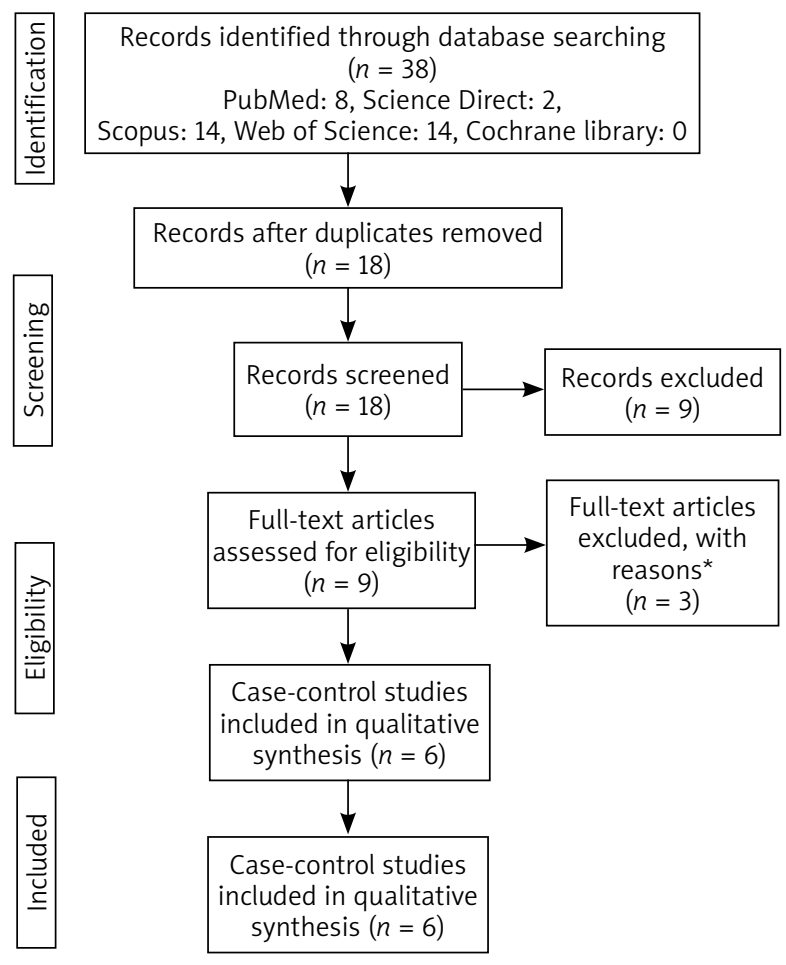

*2 studies were cross-sectional, 1 study had two intervention groups without control

Figure 1. Flowchart of the study

totoxic and allergic effects of these ions can be considered. Pazzini et al. [20] suggested that nickel can affect inflammatory reactions throughout orthodontic treatment. Therefore, considering ion levels in future studies may be necessary.

After 6-month orthodontic treatment compared with before treatment, Amini et al. [11] demonstrated that hair $\mathrm{Ni}$ and $\mathrm{Cr}$ levels increased by 381 and 16\%, respectively. Mikulewicz et al. [21] reported increases of 39 and $2.5 \%$ in $\mathrm{Ni}$ and $\mathrm{Cr}$ levels, respectively, of scalp hair after orthodontic treatment compared with before treatment, without a significant difference. Abtahi et al. [5] observed that

Table 1. Characteristics of studies included in the meta-analysis $(n=6)$

\begin{tabular}{|c|c|c|c|c|c|c|}
\hline $\begin{array}{l}\text { Reference } \\
\text { (first author, year) }\end{array}$ & Country & $\begin{array}{c}\text { No. of } \\
\text { patients }\end{array}$ & $\begin{array}{l}\text { No. of } \\
\text { controls }\end{array}$ & $\begin{array}{l}\text { Mean age [year]/ } \\
\text { male (\%) of patients }\end{array}$ & $\begin{array}{l}\text { Mean age [year]/ } \\
\text { male (\%) of controls }\end{array}$ & $\begin{array}{c}\text { Orthodontic } \\
\text { treatment period } \\
\text { [months] }\end{array}$ \\
\hline Levrini, 2006 [19] & Italy & 15 & 15 & 18.9 (range: $10-25) / 0$ & Matched/matched & - \\
\hline Mikulewicz, 2011 [16] & Poland & 28 & 18 & Matched/ matched & Matched/matched & Mean: 18-24 \\
\hline Abtahi, 2013 [5] & Iran & 24 & 24 & Range: $12-20 / 0$ & Range: $12-20 / 0$ & 4 \\
\hline Martín-Cameán, 2014 [18] & Spain & 70 & 56 & $-/ 34.3$ & $-/ 42.8$ & $\sim 24$ \\
\hline Mikulewicz, 2015 [17] & Poland & 36 & 32 & $-1-$ & $-1-$ & $\sim 69.8$ \\
\hline Jamshidi, 2018 [3] & Iran & 24 & 28 & $17.54 / 29.2$ & $15.42 / 53.8$ & 12 \\
\hline
\end{tabular}




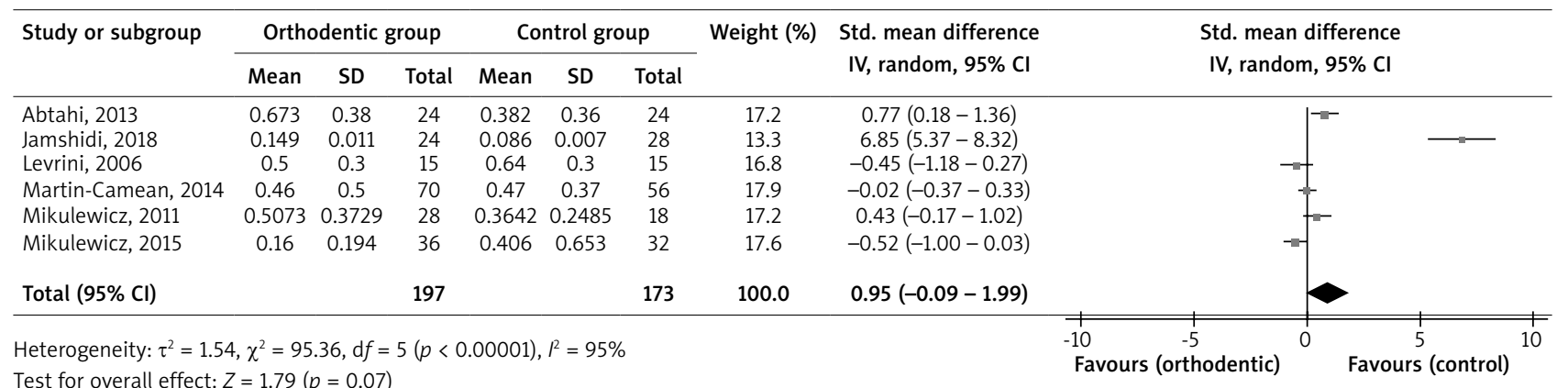

Figure 2. Forest plot of random effects of hair nickel levels in patients undergoing fixed orthodontic treatment compared with controls

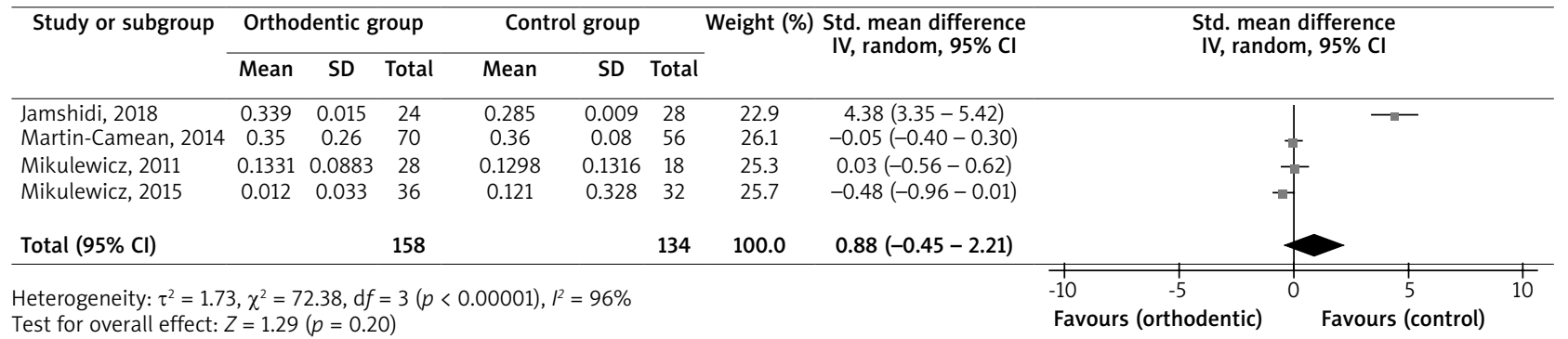

Figure 3. Forest plot of random effects of hair chromium levels in patients undergoing fixed orthodontic treatment compared with controls

Table 2. Quality of case-control studies included in the meta-analysis $(n=6)$

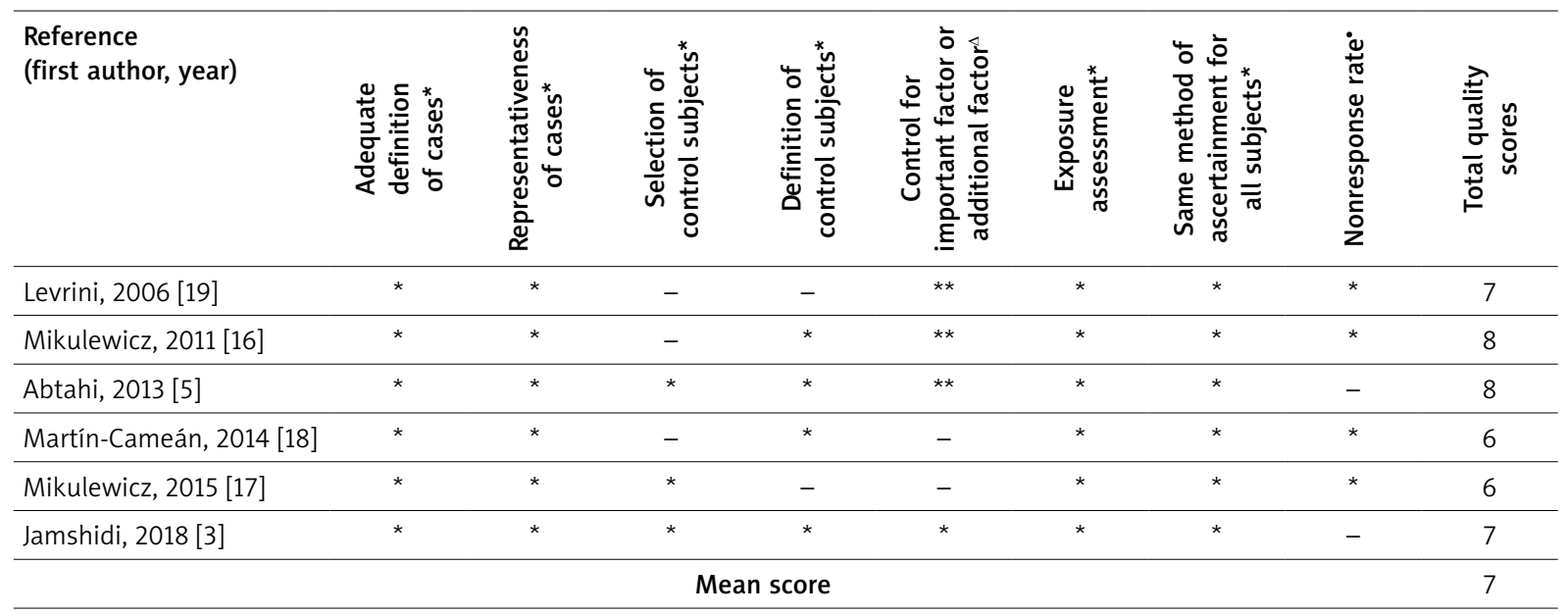

*A maximum of one star. ${ }^{\Delta}$ maximum of 2 stars, studies that matched or controlled for age received one star, whereas studies that controlled for another important factor received an additional star. ${ }^{\circ}$ One star was assigned if there was no significant difference in the response rate between control subjects and cases. 

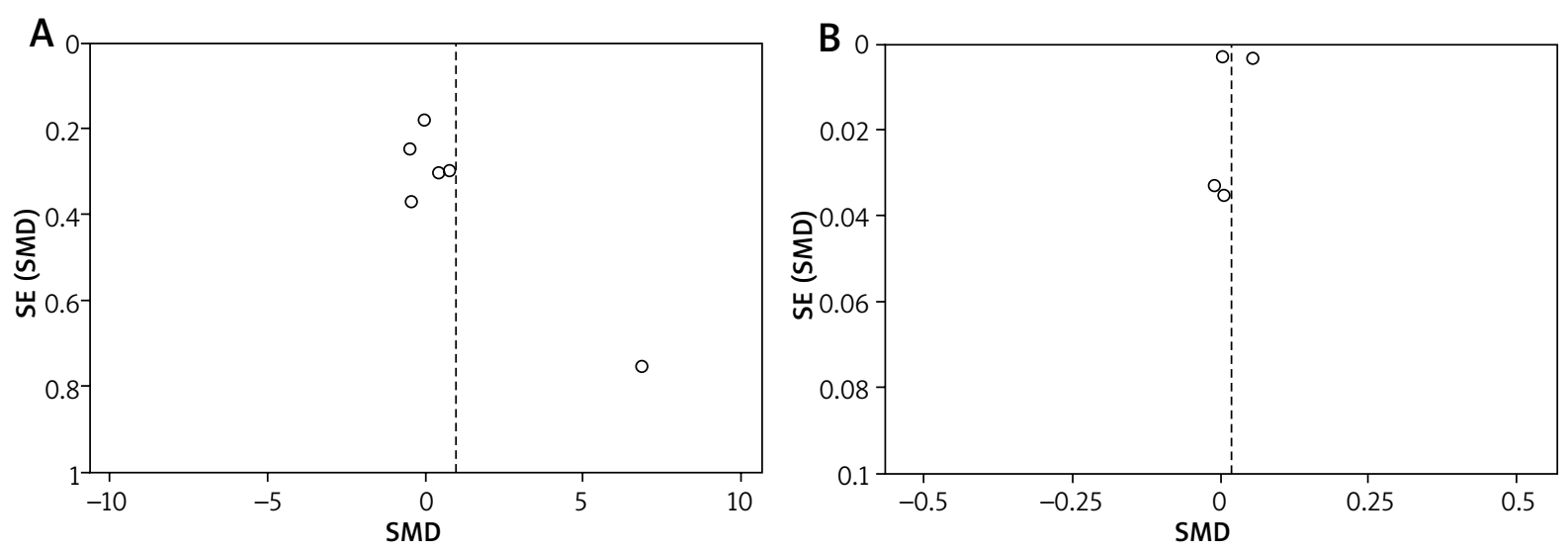

Figure 4. Funnel plot of random effect of levels of (A) hair nickel, and (B) hair chromium in patients undergoing fixed orthodontic treatment compared with controls

after 4-month treatment $\mathrm{Ni}$ changes in the scalp hair of patients undergoing fixed orthodontic treatment increased to $190 \%$. A 6-month trial with fixed orthodontic appliances consisting of either metal injection molding or conventional brackets [22] showed that hair nickel and chromium levels increased by about 200\% and 185\%, respectively. Some shampoos and many hair perm dye bleach products transfer Ni into the hair [5].

High metal ion levels in saliva were found 1 to 2 weeks after exposure to metal appliances and then these reverted to the initial levels [4, 23]. Orthodontic appliances release ions into the oral environment [24, 25] more intensely in the first months of appliance placement [24-26]. In the studies included in the meta-analysis, one study [5] after 4 months' and another study [9] after 12 months' orthodontic treatment had higher Ni levels of hair in the cases compared with the controls, whereas another study [17] after around 69.8 months' orthodontic treatment had higher levels in the controls compared with the cases. Jamshidi et al. [3] in 12 months' treatment observed higher $\mathrm{Cr}$ levels in the cases compared with the controls, whereas Mikulewicz et al. [17] after around 69.8 months' orthodontic treatment had the opposite result. Therefore, long-term treatment reduced hair levels of these ions, which may be because of reducing ions released into the oral environment. The treatment period in the study of Abtahi et al. [5] was 4 months, whereas in the study of Mikulewicz et al. [17] it was around 69.8 months, and the hair Ni level in the first study was higher than in the second study.

One study [10] evaluated the role of age and sex on hair $\mathrm{Ni}$ and $\mathrm{Cr}$ levels before orthodontic treatment that the results showed that there was no significant difference between the genders or between age $<18$ and $>18$ years. Martín-Cameán et al. [18] reported that females had higher metal levels in the cases undergoing orthodontic treatment compared with the controls, but no significant associations were found between age and metal levels in both groups.

Overall the results indicated that hair Ni level changes were greater than $\mathrm{Cr}$ level changes after treatment, especially in the subjects undergoing orthodontic treatment compared with the controls. In addition, there were different results between studies, which may be because of differences in conditions of the hair collection, treatment periods, and gender. In one study [18] the hair was washed by ultrasonic cleaning, but in another study [16] it was washed with a proprietary baby shampoo under laboratory conditions and then with water. The hair $\mathrm{Ni}$ level in the first study was less than the second study. In addition, two studies $[5,19]$ conducted the analysis on women and three other studies $[3,16,18]$ included both genders, and the results of ion analysis were different between them.

There were several significant weaknesses in the meta-analysis. First, duration of treatment and measurement methods were different in the studies. Second, age and sex were not matched between the groups. Finally, there was a low number of participants in most studies.

\section{Conclusions}

Due to the slightly elevated hair levels of $\mathrm{Ni}$ and $\mathrm{Cr}$ in the subjects undergoing fixed orthodontic therapy, changing the components of fixed orthodontic appliances can be considered as an acceptable solution in the future. Therefore, future studies need to assess the levels of these ions in serum and saliva of patients for more accurate confirmation of cytotoxic and allergic effects after fixed orthodontic therapy.

\section{Conflict of interest}

The authors declare no conflict of interest. 


\section{References}

1. Amini F, Jafari A, Amini P, Sepasi S. Metal ion release from fixed orthodontic appliances: an in vivo study. Eur J Orthod 2012; 34: 126-30.

2. Marques LS, Paiva SM, Vieira-Andrade RG, et al. Discomfort associated with fixed orthodontic appliances: determinant factors and influence on quality of life. Dental Press J Orthod 2014; 19: 102-7.

3. Jamshidi S, Rahmati Kamel M, Mirzaie M, et al. Evaluation of scalp hair nickel and chromium level changes in patients with fixed orthodontic appliance: a one-year follow-up study. Acta Odontol Scand 2018; 76: 1-5.

4. Petoumenou E, Arndt M, Keiliq L, et al. Nickel concentration in the saliva of patients with nickel-titanium orthodontic appliances. Am J Orthod Dentofacial Orthop 2009; 135: 59-65.

5. Abtahi M, Jahanbin A, Yaghoubi M, et al. Are more nickel ions accumulated in the hair of fixed orthodontic patients? Indian J Dent Res 2013; 24: 298-301.

6. Staekjaer L, Menne T. Nickel allergy and orthodontic treatment. Eur J Orthod 1990; 12: 284-9.

7. House K, Sernetz F, Dymock D, et al. Corrosion of orthodontic appliances - should we care? Am J Orthod Dentofacia Orthop 2008; 133: 584-92.

8. Shamberger RJ. Validity of hair mineral testing. Biol Trace Elem Res 2002; 87: 1-25.

9. Unkiewicz-Winiarczyk A, Bagniuk A, Gromysz-Kałkowska K, Szaubartowska E. Calcium, magnesium, iron, zinc and copper concentration in the hair of tobacco smokers. Biol Trace Elem Res 2009; 128: 152-60.

10. Mikulewicz M, Wołowiec P, Loster B, Chojnacka K. Metal ions released from fixed orthodontic appliance affect hair mineral content. Biol Trace Elem Res 2015; 163: 11-8.

11. Amini F, Mollaei M, Harandi S, et al. Effects of fixed orthodontic treatment on hair nickel and chromium levels: a 6-month prospective preliminary study. Biol Trace Elem Res 2015; 164: 12-7.

12. Moher D, Liberati A, Tetzlaff J, Altman DG; PRISMA Group. Preferred reporting items for systematic reviews and meta-analyses: the PRISMA statement. PLoS Med 2009; 6: e1000097.

13. Wells GA, Shea B, O'Connell D, et al. The Newcastle-Ottawa scale (NOS) for assessing the quality of non-randomised studies in meta-analyses. Ottawa: Ottawa Hospital Research Institute; 2011. http://www.ohri.ca/programs/clinical_epidemiology/oxford.asp. Accessed $12 \operatorname{Jan} 2016$.

14. Higgins JP, Thompson SG. Quantifying heterogeneity in a meta-analysis. Stat Med 2002; 21: 1539-58.

15. Egger M, Davey Smith G, Schneider M, et al. Bias in metaanalysis detected by a simple, graphical test. BMJ 1997; 315: 629-34.

16. Mikulewicz M, Chojnacka K, Zielińska A, Michalak I. Exposure to metals from orthodontic appliances by hair mineral analysis. Environ Toxicol Pharmacol 2011; 32: 10-6.

17. Mikulewicz M, Kachniarz K, Chojnacka K. Exposure of cleft lip and palate patients to toxic elements released during orthodontic treatment in the study of non-invasive matrices. PLoS One 2015; 10: e0140211.

18. Martín-Cameán A, Molina-Villalba I, Jos A, et al. Biomonitorization of chromium, copper, iron, manganese and nickel in scalp hair from orthodontic patients by atomic absorption spectrometry. Environ Toxicol Pharmacol 2014; 37: 759-71.

19. Levrini L, Lusvardi G, Gentile D. Nickel ions release in patients with fixed orthodontic appliances. Minerva Stomatol 2006; 55: 115-21.
20. Pazzini CA, Júnior GO, Marques LS, et al. Prevalence of nickel allergy and longitudinal evaluation of periodontal abnormalities in orthodontic allergic patients. Angle Orthod 2009; 79: 922-7.

21. Mikulewicz M, Chojnacka K. Release of metal ions from orthodontic appliances by in vitro studies: a systematic literature review. Biol Trace Elem Res 2011; 139: 241-56.

22. Masjedi MK, Jahromi NH, Niknam O, et al. Effects of fixed orthodontic treatment using conventional (two-piece) versus metal injection moulding brackets on hair nickel and chromium levels: a double-blind randomized clinical trial. Eur J Orthod 2017; 39: 17-24.

23. Ağaoğlu G, Arun T, Izgi B, Yarat A. Nickel and chromium levels in the saliva and serum of patients with fixed orthodontic appliances. Angle Orthod 2001; 71: 375-9.

24. Gölz L, Knickenberg AC, Keilig L, et al. Nickel ion concentrations in the saliva of patients treated with self-ligating fixed appliances: a prospective cohort study. J Orofac Orthop 2016; 77: 85-93.

25. Khaneh Masjedi M, Niknam O, Haghighat Jahromi N, et al. Effects of fixed orthodontic treatment using conventional, copper-included, and epoxy-coated nickel-titanium archwires on salivary nickel levels: a double-blind randomized clinical trial. Biol Trace Elem Res 2016; 174: 27-31.

26. Sahoo N, Kailasam V, Padmanabhan S, Chitharanjan AB. In-vivo evaluation of salivary nickel and chromium levels in conventional and self-ligating brackets. Am J Orthod Dentofacial Orthop 2011; 140: 340-5. 